\title{
ON THE HOCHSCHILD HOMOLOGY OF SMASH BIPRODUCTS
}

\author{
A. KAYGUN AND S. SÜTLÜ
}

\begin{abstract}
Aвstract. We develop a new spectral sequence in order to calculate Hochschild homology of smash biproducts (also called twisted tensor products) of unital associative algebras $A \# B$ provided one of $A$ or $B$ has Hochschild dimension less than 2. We use this spectral sequence to calculate Hochschild homology of quantum tori, multiparametric quantum affine spaces, quantum complete intersections, quantum Weyl algebras, deformed completed Weyl algebras, and finally the algebra $M_{q}(2)$ of quantum $2 \times 2$-matrices.
\end{abstract}

\section{INTRODUCTION}

In this paper we investigate the Hochschild homology of a class of product algebras called the smash biproducts [5] (or also referred as the twisted tensor products [6]) that include Hopf-cross products and Ore extensions. Then we effectively calculate Hochschild homologies of quantum tori, multiparametric (quantum) affine spaces [16, 30], quantum complete intersections [3], quantum Weyl algebras [26], deformed completed Weyl algebras [9, 21], and the quantum matrix algebra $M_{q}(2)$ [1, 18].

Our strategy relies on splitting the Hochschild complex into a (twisted) product of two Hochschild complexes induced by the decomposition of the underlying algebra. Then we combine the homologies of the individual pieces via a suitable spectral sequence. In doing so, we do not rely on any ad-hoc resolutions of the underlying algebras as bimodules over themselves. The key observation is that if one of the component algebras has Hochschild homological dimension less than 2, then the spectral sequence degenerates on the $E^{2}$-page yielding the result immediately. We refer the reader to Section 2 for technical details.

Here is a plan of the paper: In Section 1 we recall basic definitions and tools needed to work with smash biproduct algebras, and then in Section 2 we construct our homological machinery. Section 3 contains our calculations. In Section 3.3 we calculate the Hochschild homologies of the quantum plane $k[x] \# k[y]$, the quantum cylinder $k[x] \# k\left[y, y^{-1}\right]$, and the quantum torus $k\left[x, x^{-1}\right] \# k\left[y, y^{-1}\right]$. Next, in Section 3.4 we calculate the Hochschild homology of the multiparametric affine space $S\left(X_{v}, \Lambda\right)$ [16], as well as a particular case of quantum Weyl algebras [26]. The Hochschild homology of the quantum complete intersection algebra $C_{a, b}:=k\langle x, y\rangle /\left\langle x^{a}, x y-q y x, y^{b}\right\rangle$ for every $a, b \geqslant 2[3]$ is computed in Section 3.5. In Section 3.7. we deal with the quantum Weyl algebra $S\left(X_{\mu}, Y_{\nu}, \Lambda\right)[26]$ in full generality. Subsection 3.6 deals with the $(\mathfrak{g}, D)$-deformed Weyl algebra $A_{\mathfrak{q}, D}^{\mathrm{pol}}:=S(V) \rtimes U(\mathfrak{g})$, and its completion $A_{\mathfrak{g}, D}:=\widehat{S(V)} \rtimes U(\mathfrak{g})[9,21]$. Although deformed Weyl algebras are Hopf-cross products, neither component is necessarily of low Hochschild homological dimension. Nevertheless, our method plays a critical role in identifying the (continuous) Hochschild homology of $A_{\mathfrak{g}, D}$ with its dense subalgebra $A_{\mathfrak{a}, D}^{\mathrm{pol}}$. In particular, we obtain the results [12, Thm. 2.1] and [13, Thm. 4] for the for the completed Weyl algebra $A_{2 n}$, since the Hochschild homology of $A_{2 n}^{\mathrm{pol}}$ is already known. The last subsection, Subsection 3.8, is devoted to the Hochschild homology of the quantum matrix algebra $M_{q}(2)$. The fact that the algebra $M_{q}(2)$ is a short tower of Ore extensions [18] makes it amenable for the tools we develop here. In fact, all quantum matrix algebras $M_{q}(n)$ are towers of Ore extensions [1, 15, 18]. However, the growth of the lengths of these towers renders the arguments we use in the present paper ineffective. As such, the homology of the algebras $M_{q}(n)$ for $n>2$, and the quantum linear groups [23] demand a different approach, which we postpone to a subsequent paper. 
Notation and Conventions. We fix a ground field $k$ of characteristic 0 . All algebras are assumed to be unital and associative, but not necessarily finite dimensional. We use $k[S]$ and $k\{S\}$ to denote respectively the free commutative and free noncommutative polynomial algebras generated by a set $S$. We also use $\langle S\rangle$ to denote either the $k$-vector space spanned, or the two-sided ideal, generated by the set $S$ depending on the context. If $G$ is a group, $k[G]$ denotes the group algebra of $G$ over $k$.

\section{Smash biproducts, Hopf-Cross Products, and Ore eXtensions}

\subsection{The smash biproduct of algebras.}

Let us now recall from [5] the smash biproduct of algebras, which are also called the twisted tensor products [6]. Let $A$ and $B$ be two algebras, and let $R: B \otimes A \longrightarrow A \otimes B$ be a linear map written as $R(b \otimes a)=:{ }^{R} a \otimes b^{R}$ making the diagrams
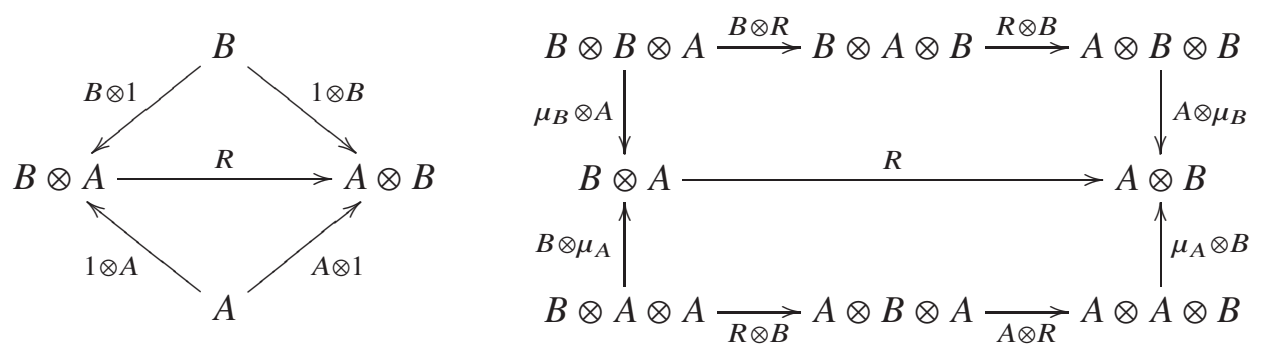

commutative. We shall call any such map a distributive law. One can then show that $A \otimes B$ is a unital associative algebra with the multiplication

$$
(a \otimes b)\left(a^{\prime} \otimes b^{\prime}\right):=a\left({ }^{R} a^{\prime}\right) \otimes\left(b^{R}\right) b^{\prime},
$$

for any $a, a^{\prime} \in A$ and $b, b^{\prime} \in B$, and with the unit $1 \otimes 1 \in A \otimes B$, if and only if the diagrams in (1.1) commute, [5, Thm. 2.5]. We will use $A \#_{R} B$ to denote this algebra.

Let us note that the natural inclusions

$$
i_{A}: A \longrightarrow A \#_{R} B, \quad a \mapsto a \otimes 1, \quad i_{B}: B \longrightarrow A_{R} B, \quad b \mapsto 1 \otimes b
$$

are algebra maps, and that the map

$$
\Psi:=\mu_{A \#_{R} B} \circ\left(i_{A} \otimes i_{B}\right): A \otimes B \longrightarrow A \#_{R} B
$$

is an isomorphism of vector spaces. Then, the map $R$ can be recovered as

$$
R=\Psi^{-1} \circ \mu_{A \#_{R} B} \circ\left(i_{B} \otimes i_{A}\right) .
$$

Let us next review a few (more concrete) examples of this construction.

\subsection{Ore extensions.}

Let $A$ be an algebra, and $k[X]$ be the polynomial algebra in one indeterminate. Let $\alpha: A \rightarrow A$ be an algebra homomorphism, and $\delta: A \rightarrow A$ be a derivation. Then the invertible distributive law defined as

$$
R: k[X] \otimes A \longrightarrow A \otimes k[X], \quad R(X \otimes a):=\alpha(a) \otimes X+\delta(a) \otimes 1
$$

yields the Ore extension associated to the datum $(A, \alpha, \delta)$, i.e. $A \#_{R} k[X] \cong A[X, \alpha, \delta]$, [5, Ex. 2.11].

We refer the reader to [25, 18] for an account of the Ore extensions in the quantum group theory. 


\subsection{Hopf-cross products.}

We note from [5, Ex. 4.2] that the smash biproduct construction covers Hopf-cross products, i.e. algebras of the form $A \otimes H$ where $H$ is a Hopf algebra and $A$ is a (left) $H$-module algebra. The latter means that there is an action $\triangleright: H \otimes A \rightarrow A$ which satisfies

$$
h \triangleright(a b)=\left(h_{(1)} \triangleright a\right)\left(h_{(2)} \triangleright b\right)
$$

for any $h \in H$, and any $a, b \in A$. Then, there is an algebra structure on $A \rtimes H:=A \otimes H$ given by

$$
(a \otimes h)(b \otimes g)=a\left(h_{(1)} \triangleright b\right) \otimes h_{(2)} g,
$$

for any $a, b \in A$, and any $h, g \in H$, with unit $1 \otimes 1 \in A \rtimes H$. Now letting

$$
R: H \otimes A \rightarrow A \otimes H, \quad R(h \otimes a)=h_{(1)} \triangleright a \otimes h_{(2)}
$$

for any $a \in A$ and any $h \in H$ it follows from (1.2) that $A \rtimes H=A \#_{R} H$.

\subsection{Algebras with automorphisms.}

Let $A$ be any unital associative algebra, and let $G$ be a group acting on $A$ via automorphisms. Consider the distributive law $R: k[G] \otimes A \rightarrow A \otimes k[G]$ given by

$$
R(g \otimes a)=(g \triangleright a) \otimes g
$$

for every $a \in A$ and $g \in G$. Let us denote the smash biproduct algebra $A \#_{R} k[G]$ simply by $A \# G$. This is a special case of the smash product given by the distributive law (1.3) for the Hopf algebra $H=k[G]$.

\subsection{Algebras with derivations.}

Let $\mathfrak{g}$ be a Lie algebra, and $A$ a unital associative algebra on which $\mathfrak{g}$ acts by derivations; that is,

$$
X \triangleright(a b)=(X \triangleright a) b+a(X \triangleright b)
$$

for any $a, b \in A$, and any $X \in \mathfrak{g}$. Then,

$$
R: U(\mathfrak{g}) \otimes A \rightarrow A \otimes U(\mathfrak{g}), \quad R(X \otimes a)=(X \triangleright a) \otimes 1+a \otimes X
$$

determines an invertible distributive law, and hence the smashed biproduct algebra $A \#_{R} U(\mathfrak{g})$ which we simply denote by $A \#$ g.

Let us note that the above conditions endows $A$ with a $U(\mathfrak{g})$-module algebra structure, that is, the algebra $A \# \mathfrak{g}$ is a special case of the Hopf crossed product $A \rtimes H$ with $H=U(\mathfrak{g})$, and the distributive law (1.3).

\section{HoMOlOGY OF SMASH BIPRODUCTS}

\subsection{The bar complex.}

Given an associative algebra $A$, together with a right $A$-module $V$ and a left $A$-module $W$, one can form the two sided bar complex

$$
\mathrm{CB}_{*}(V, A, W):=\bigoplus_{n \geqslant 0} V \otimes A^{\otimes n} \otimes W
$$

together with the differentials $d: \mathrm{CB}_{n}(V, A, W) \longrightarrow \mathrm{CB}_{n-1}(V, A, W), n \geqslant 1$, defined as

$$
\begin{aligned}
d\left(v \otimes a_{1} \otimes \cdots \otimes a_{n} \otimes w\right) & =v \cdot a_{1} \otimes a_{2} \otimes \cdots \otimes a_{n} \otimes w \\
& +\sum_{j=1}^{n-1}(-1)^{j} v \otimes \cdots \otimes a_{j} a_{j+1} \otimes \cdots \otimes w \\
& +(-1)^{n} v \otimes a_{1} \otimes \cdots \otimes a_{n-1} \otimes a_{n} \cdot w .
\end{aligned}
$$




\subsection{Hochschild homology.}

Now, let $A$ be a $k$-algebra and $V$ be an $A$-bimodule, or equivalently, a right module over the enveloping algebra $A^{e}:=A \otimes A^{\mathrm{op}}$. Then the Hochschild homology of $A$, with coefficients in $V$, is defined to be

$$
H_{*}(A, V):=\operatorname{Tor}_{*}^{A^{e}}(A, V) .
$$

Equivalently, see for instance [20, Prop. 1.1.13], the Hochschild homology of $A$, with coefficients in the $A$-bimodule $V$, is the homology of the complex

$$
\mathrm{CH}_{*}(A, V):=\bigoplus_{n \geqslant 0} \mathrm{CH}_{n}(A, V), \quad \mathrm{CH}_{n}(A, V):=V \otimes A^{\otimes n}
$$

with respect to the differential $b: \mathrm{CH}_{n}(A, V) \longrightarrow \mathrm{CH}_{n-1}(A, V)$, defined for $n \geqslant 1$ as

$$
\begin{aligned}
b\left(v \otimes a_{1} \otimes \cdots \otimes a_{n}\right) & =v \cdot a_{1} \otimes a_{2} \otimes \cdots \otimes a_{n} \\
& +\sum_{j=1}^{n-1}(-1)^{j} v \otimes a_{1} \otimes \cdots \otimes a_{j} a_{j+1} \otimes \cdots \otimes a_{n} \\
& +(-1)^{n} a_{n} \cdot v \otimes a_{1} \otimes \cdots \otimes a_{n-1} .
\end{aligned}
$$

\subsection{Hochschild homology with invertible distributive laws.}

Let $R: B \otimes A \rightarrow A \otimes B$ be an invertible distributive law, and $V$ an $A \#_{R} B$-bimodule, that is, the diagram

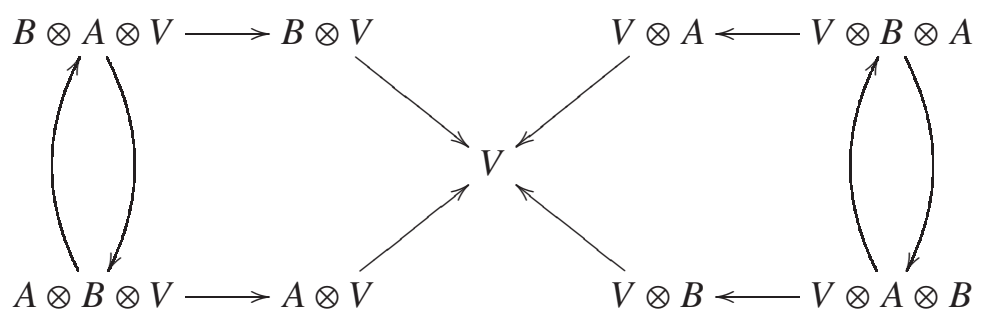

is commutative. We now introduce a bisimplicial complex computing the Hochschild homology, with coefficients, of the algebra $A \#_{R} B$. We shall need an iterative use of the distributive law (and its inverse), as such, we make use of the notation

$$
R\left(b_{j} \otimes a_{i}\right)={ }^{R_{j i}} a_{i} \otimes b_{j}^{R_{j i}}, \quad R^{-1}\left(a_{i} \otimes b_{j}\right)={ }^{L_{j i}} b_{j} \otimes a_{i}^{L_{j i}} .
$$

Proposition 2.1. Given a smash biproduct algebra $A_{R} B$ with an invertible distributive law $R: B \otimes A \rightarrow$ $A \otimes B$, and an $A \#_{R} B$-bimodule $V$, the isomorphism

$$
\begin{aligned}
v \otimes\left(a_{1}, b_{1}\right) \otimes \cdots \otimes\left(a_{n}, b_{n}\right) & \mapsto \\
& a_{1}^{L_{11} \ldots L_{n 1}} \otimes \cdots \otimes a_{n-1}^{L_{(n-1)(n-1)} L_{(n-1) n}} \otimes a_{n}^{L_{n n}} \otimes v \otimes{ }^{L_{11}} b_{1} \otimes{ }^{L_{21} L_{22}} b_{2} \otimes \cdots \otimes \otimes^{L_{n 1} \ldots L_{n n}} b_{n}
\end{aligned}
$$

identifies the Hochschild homology complex of the algebra $A \#_{R} B$, with coefficients in $V$, with the diagonal of the bisimplicial complex given by $\mathcal{B}_{p, q}(A, V, B):=A^{\otimes p} \otimes V \otimes B^{\otimes q}$ with the structure maps

$$
\begin{aligned}
& \partial_{i}^{h}\left(a_{1} \otimes \cdots \otimes a_{q} \otimes v \otimes b_{1} \otimes \cdots \otimes b_{p}\right) \\
& := \begin{cases}a_{1} \otimes \cdots \otimes a_{q} \otimes v \triangleleft b_{1} \otimes \cdots \otimes b_{p} & \text { if } i=0, \\
a_{1} \otimes \cdots \otimes a_{q} \otimes v \otimes b_{1} \otimes \cdots \otimes b_{i} b_{i+1} \otimes \cdots \otimes b_{p} & \text { if } 1 \leqslant i \leqslant p-1, \\
R_{p 1} a_{1} \otimes \cdots \otimes{ }_{p q} a_{q} \otimes b_{p}^{R_{p 1} \cdots R_{p q}} \triangleright v \otimes b_{1} \otimes \cdots \otimes b_{p-1} & \text { if } i=p,\end{cases} \\
& \partial_{j}^{v}\left(a_{1} \otimes \cdots \otimes a_{q} \otimes v \otimes b_{1} \otimes \cdots \otimes b_{p}\right) \\
& := \begin{cases}a_{2} \otimes \cdots \otimes a_{q} \otimes v \triangleleft R_{11} \cdots R_{p 1} a_{1} \otimes b_{1}^{R_{11}} \otimes \cdots \otimes b_{p}^{R_{p 1}} & \text { if } j=0, \\
a_{1} \otimes \cdots \otimes a_{j} a_{j+1} \otimes a_{q} \otimes \cdots \otimes v \otimes b_{1} \otimes \cdots \otimes b_{p} & \text { if } 1 \leqslant j \leqslant q-1, \\
a_{1} \otimes \cdots \otimes a_{q-1} \otimes a_{q} \triangleright v \otimes b_{1} \otimes \cdots \otimes b_{p} & \text { if } j=q .\end{cases}
\end{aligned}
$$


Proof. We observe that

$$
\begin{aligned}
\partial_{p}^{h} \partial_{0}^{v}\left(a_{1} \otimes \cdots \otimes a_{q} \otimes v \otimes b_{1} \otimes \cdots \otimes b_{p}\right) \\
\quad=\partial_{p}^{h}\left(a_{2} \otimes \cdots \otimes a_{q} \otimes v \triangleleft R_{11} \cdots R_{p 1} a_{1} \otimes b_{1}^{R_{11}} \otimes \cdots \otimes b_{p}^{R_{p 1}}\right) \\
\quad={ }^{R_{p 2}} a_{2} \otimes \cdots \otimes{ }^{R_{p q}} a_{q} \otimes b_{p}^{R_{p 1} \cdots R_{p q}} \triangleright\left(v \triangleleft{ }^{R_{11} \ldots R_{p 1}} a_{1}\right) \otimes b_{1}^{R_{11}} \otimes \cdots \otimes b_{p-1}^{R_{(p-1) 1}} \\
\quad={ }^{R_{p 2}} a_{2} \otimes \cdots \otimes{ }^{R_{p q}} a_{q} \otimes\left(b_{p}^{R_{p 1} \ldots R_{p q}} \triangleright v\right) \triangleleft{ }^{R_{11} \cdots R_{p 1}} a_{1} \otimes b_{1}^{R_{11}} \otimes \cdots \otimes b_{p-1}^{R_{(p-1) 1}} \\
\quad=\partial_{0}^{v}\left({ }^{R_{p 1}} a_{1} \otimes \cdots \otimes{ }^{R_{p q}} a_{q} \otimes b_{p}^{R_{p 1} \cdots R_{p q}} \triangleright v \otimes b_{1} \otimes \cdots \otimes b_{p-1}\right) \\
=\partial_{0}^{v} \partial_{p}^{h}\left(a_{1} \otimes \cdots \otimes a_{q} \otimes v \otimes b_{1} \otimes \cdots \otimes b_{p}\right) .
\end{aligned}
$$

For $1 \leqslant i \leqslant p-1$ and $1 \leqslant j \leqslant q-1$, the commutativity $\partial_{i}^{h} \partial_{j}^{v}=\partial_{j}^{v} \partial_{i}^{h}$ follows from the commutativity of the second diagram in 1.1.

Considering the spectral sequence associated to the filtration by the rows (or the columns) of the bicomplex given in Proposition 2.1, we obtain the following result.

Theorem 2.2. Let $R: B \otimes A \rightarrow A \otimes B$ be an invertible distributive law, and let $V$ be an $A \#_{R} B$-bimodule. Then there are two spectral sequences whose $E^{1}$-terms are given by

$$
E_{p, q}^{1}=H_{q}\left(A, \mathrm{CH}_{p}(B, V)\right), \quad \quad{ }^{\prime} E_{p, q}^{1}=H_{p}\left(B, \mathrm{CH}_{q}(A, V)\right)
$$

which converge to the Hochschild homology of the smash biproduct $A \#_{R} B$, with coefficients in $V$.

Corollary 2.3. Let $\mathrm{g}$ be a Lie algebra action on an algebra A by derivations, and let $G$ be a group action on an algebra B by automorphisms. Let also $V$ be an $A \# g$-bimodule, and $W$ a B\#G-bimodule. Then there are two spectral sequences such that

$$
H_{p+q}(A \# \mathfrak{g}, V) \Leftarrow{ }^{\prime} E_{p, q}^{1}=H_{p}\left(\mathfrak{g}, \mathrm{CH}_{q}(A, V)^{a d}\right),
$$

and

$$
H_{p+q}(B \# G, W) \Leftarrow{ }^{\prime} E_{p, q}^{1}=H_{p}\left(G, \mathrm{CH}_{q}(B, W)^{a d}\right) .
$$

\subsection{Homology of smash biproducts by amenable algebras.}

We will call a unital associative algebra $B$ as amenable if it has Hochschild homological dimension 0 , in other words $B$ is a $B^{e}$-flat module [17]. In the discrete (algebraic) case, the typical examples are group algebras $k[G]$ of finite groups where the characteristic of the field $k$ does not divide the order of the group $G$. In the measurable case, as in the case dealt originally in [17], the typical examples are of the form $L^{1}(G)$ where $G$ is an amenable group. In particular, all compact groups and locally compact abelian groups are amenable, [24, Chap.3].

In the sequel, we make frequent use of the notation

$$
V_{B}:=\frac{V}{[B, V]}=\frac{V}{\langle b v-v b \mid v \in V, b \in B\rangle} \cong H_{0}(B, V)
$$

and

$$
V^{B}:=\{v \in V \mid b v=v b, \text { for all } b \in B\} \cong H^{0}(B, V)
$$

for every $B$-bimodule $V$.

Theorem 2.4. Let $A$ and $B$ be two unital associative algebras, where $B$ is amenable, and let $R: B \otimes A \rightarrow$ $A \otimes B$ be an invertible distributive law. Then,

$$
H_{n}\left(A \#_{R} B, V\right) \cong H_{n}(A, V)_{B}
$$

for any $n \geqslant 0$, and for any $A \#_{R} B$-bimodule $V$. 
Proof. By Theorem 2.2 we have

$$
H_{*}\left(A \#_{R} B, V\right) \Leftarrow{ }^{\prime} E_{p, q}^{1}=H_{p}\left(B, \mathrm{CH}_{q}(A, V)\right) .
$$

Since $B$ is amenable, we have

$$
{ }^{\prime} E_{p, q}^{1} \cong H_{p}\left(B, \mathrm{CH}_{q}(A, V)\right) \cong \begin{cases}\mathrm{CH}_{q}(A, V)_{B} & \text { if } p=0, \\ 0 & \text { otherwise. }\end{cases}
$$

Furthermore, since $B$ is flat as a left $B^{e}$-module, the functor $(\cdot) \otimes_{B^{e}} B$ is exact. As such,

$$
H_{*}\left(A \#_{R} B, V\right) \Leftarrow{ }^{\prime} E_{p, q}^{2}= \begin{cases}H_{q}(A, V)_{B} & \text { if } p=0, \\ 0 & \text { otherwise. }\end{cases}
$$

The result then follows from the spectral sequence consisting of only one column.

In particular, for $V=A$ and $B=k[G]$, where $G$ is a finite group, we have the following.

Corollary 2.5. Let $G$ be a finite group acting on a unital associative algebra A by automorphisms. Then

$$
H_{*}(A \# G, A) \cong H H_{*}(A)_{k G} \cong H H_{*}(A)^{G},
$$

where $H H_{*}(A)^{G}$ denotes the space of $G$-invariants (under the diagonal action).

\subsection{Homology of smash biproducts by smooth algebras.}

A unital associative $k$-algebra $B$ is called smooth if $B$ has Hochschild cohomological dimension 1, i.e. the kernel of the multiplication map $\mu_{B}: B \otimes B \rightarrow B$ is $B^{e}$-projective; [27, Lemma 2.3]. Such algebras are also referred as quasi-free; [7]. Among the most basic examples of smooth algebras, there are $k[x]$ and $k\left[x, x^{-1}\right]$, see [20, Ex. 3.4.3]. Similarly, we call an algebra $m$-smooth if $\Omega_{B \mid k}$ has Hochschild homological dimension $m$. As such, an ordinary smooth algebra is 0 -smooth. In [29], a smooth algebra is defined to an algebra $B$ whose projective $B^{e}$-resolution is finite, and contains only finitely generated $B^{e}$-modules. The quintessential example is $B=S(V)$; the polynomial algebra with $m+1=\operatorname{dim}_{k}(V)$-indeterminates which is $m$-smooth.

Lemma 2.6. Let $B$ be a smooth algebra, and $V$ a B-bimodule. Then,

$$
H_{1}(B, V) \cong V^{B} \cong H^{0}(B, V) \text {. }
$$

Proof. Since $B$ is smooth

$$
0 \rightarrow \operatorname{ker}\left(\mu_{B}\right) \rightarrow B \otimes B \rightarrow B \rightarrow 0
$$

is a $B^{e}$-projective resolution of $B$. Then we immediately see that

$$
H_{1}(B, V) \cong \operatorname{ker}\left(V \otimes_{B^{e}} \operatorname{ker}\left(\mu_{B}\right) \rightarrow V\right) .
$$

The claim then follows from $\operatorname{ker}\left(\mu_{B}\right)$ being generated, as a $B^{e}$-module, by the elements of the form $1 \otimes x-x \otimes 1 \in B^{e}$.

Theorem 2.7. Let $A$ and $B$ be two algebras with $B$ being smooth, and let $R: B \otimes A \rightarrow A \otimes B$ be an invertible distributive law. Then, for any $A \#_{R} B$-bimodule $V$,

$$
H_{n}\left(A \#_{R} B, V\right) \cong H_{n}\left(\mathrm{CH}_{*}(A, V)_{B}\right) \oplus H_{n-1}\left(\mathrm{CH}_{*}(A, V)^{B}\right)
$$

for all $n \geqslant 0$.

Proof. In view of the smoothness of $B$, Theorem 2.2 implies that the 1 st page ' $E_{p, q}^{1}$ of the spectral sequence of Theorem 2.2 consists of two columns at $p=0$ and $p=1$. Then by (2.8)

$$
{ }^{\prime} E_{0, q}^{2}=H_{q}\left(H_{0}\left(B, \mathrm{CH}_{*}(A, V)\right)\right) \cong H_{q}\left(\mathrm{CH}_{*}(A, V)_{B}\right),
$$

and by Lemma 2.6

$$
{ }^{\prime} E_{1, q}^{2}=H_{q}\left(H_{1}\left(B, \mathrm{CH}_{*}(A, V)\right)\right) \cong H_{q}\left(\mathrm{CH}_{*}(A, V)^{B}\right)
$$

as we wanted to show. 


\section{Computations}

\subsection{Galois extensions.}

Let $K / k$ be a finite Galois extension, and let $G$ be the Galois group of this extension. Accordingly, we have the $K$-algebra $K \# G$, and from Theorem 2.4 we obtain

$$
H_{n}(K \# G, K)=H_{n}(K)^{G} .
$$

The homology of $K$, on the other hand, is given by

$$
H H_{n}(K)= \begin{cases}K & \text { if } n=0, \\ 0 & \text { otherwise }\end{cases}
$$

when regarded as a $K$-algebra. Finally $G$ being the Galois group of the extension $K / k$, we have $K^{G}=k$. Hence,

$$
H_{n}(K \# G, K)= \begin{cases}k & \text { if } n=0, \\ 0 & \text { otherwise }\end{cases}
$$

\subsection{Group rings with faithful characters.}

Let $G$ be a group and let $\sigma: G \rightarrow k^{\times}$be a character. Let us define

$$
R: k\left[x, x^{-1}\right] \otimes k[G] \rightarrow k[G] \otimes k\left[x, x^{-1}\right], \quad R\left(x^{n} \otimes g\right):=\sigma(g)^{n} g \otimes x^{n}
$$

for any $n \geqslant 0$, and any $g \in G$. Then (3.1) obeys the diagram (1.1), and we have the smash biproduct algebra $k[G] \# k\left[x, x^{-1}\right]$. Now, since $k\left[x, x^{-1}\right]$ is smooth, we have by Theorem 2.7

$$
\begin{aligned}
H_{n}\left(k[G] \# k\left[x, x^{-1}\right]\right) \cong & H_{n}\left(\mathrm{CH}_{*}\left(k[G], k[G] \# k\left[x, x^{-1}\right]\right)_{k\left[x, x^{-1}\right]}\right) \\
& \oplus H_{n-1}\left(\mathrm{CH}_{*}\left(k[G], k[G] \# k\left[x, x^{-1}\right]\right)^{k\left[x, x^{-1}\right]}\right) .
\end{aligned}
$$

On the other hand,

$$
\left[x, g_{1} \otimes \cdots \otimes g_{n} \otimes\left(g_{0} \otimes x^{\ell-1}\right)\right]=\left(\sigma\left(g_{0} g_{1} \cdots g_{n}\right)-1\right) g_{1} \otimes \cdots \otimes g_{n} \otimes\left(g_{0} \otimes x^{\ell}\right)
$$

Now, assume $\sigma$ is faithful, i.e. $\sigma(g)=1$ if and only if $g=e$. In view of the faithfulness of the character we get

whereas

$$
\mathrm{CH}_{*}\left(k[G], k[G] \# k\left[x, x^{-1}\right]\right)_{k\left[x, x^{-1}\right]}=0,
$$

$$
\mathrm{CH}_{*}\left(k[G], k[G] \# k\left[x, x^{-1}\right]\right)^{k\left[x, x^{-1}\right]} \cong \mathrm{CH}_{*}^{(e)}(k[G]) \otimes k\left[x, x^{-1}\right]
$$

where

$$
\mathrm{CH}_{n}^{(e)}(k[G]):=\left\langle g_{1} \otimes \cdots \otimes g_{n} \otimes g_{0} \in \mathrm{CH}_{n}(k[G]) \mid g_{1} \cdots g_{n} g_{0}=e\right\rangle .
$$

In order to compute the homology of this subcomplex we shall need the following lemma which goes back to Eilenberg and MacLane [10]. (See [28] for a history of Hochschild (co)homology of group algebras.)

Lemma 3.1. Let $G$ be an arbitrary group and consider $\mathrm{CH}_{*}(k[G])$. Now, consider the subcomplex $\mathrm{CH}_{*}^{(e)}(k[G])$ generated by homogeneous tensors of the form $g_{0} \otimes \cdots \otimes g_{n}$ such that the product $g_{0} \cdots g_{n}$ is the unit element. Then

$$
H_{*}\left(\mathrm{CH}_{*}^{(e)}(k[G]) \cong H_{*}(k[G], k) .\right.
$$

Proof. The morphism of complexes $\mathrm{CH}_{n}(k[G], k) \rightarrow \mathrm{CH}_{n}^{(e)}(k[G], k[G])$, given by

$$
g_{1} \otimes \cdots \otimes g_{n} \mapsto g_{1} \otimes \cdots \otimes g_{n} \otimes g_{n}^{-1} \cdots g_{1}^{-1},
$$

is an isomorphism. 
Accordingly, we have

$$
H_{*}\left(\mathrm{CH}_{*}\left(k[G], k[G] \# k\left[x, x^{-1}\right]\right)^{k\left[x, x^{-1}\right]}\right) \cong H_{*}(k[G], k) \otimes k\left[x, x^{-1}\right],
$$

and hence

$$
H H_{n}\left(k[G] \# k\left[x, x^{-1}\right]\right) \cong H_{n-1}(G, k) \otimes k\left[x, x^{-1}\right] .
$$

Let $G$ be a finitely generated abelian group of the form $G=G^{f} \times G^{t}$ where $G^{t}$ is the maximal torsion subgroup and $G^{f}$ is the maximal torsion-free subgroup of $G$. Since $H_{n}\left(G^{t}, k\right)=0$ for $n \geqslant 1$, we see that the higher homology is determined $G^{f}$. Let $a$ be the free rank of $G$. Then

$$
H H_{n}\left(k[G] \# k\left[x, x^{-1}\right]\right) \cong k^{\left({ }_{n-1}^{a}\right)} \otimes k\left[x, x^{-1}\right] .
$$

\subsection{Smash products of Lorentz polynomials.}

Below, we shall consider the Hochschild homology of the smash products of polynomial algebra $k[x]$ and the Laurent polynomial algebra $k\left[x, x^{-1}\right]$ in various combinations, but always with the particular distributive law given by

$$
R\left(y^{j} \otimes x^{i}\right)=q^{i j} x^{i} \otimes y^{j}
$$

for some fixed $q \in k^{\times}$which is not a root of unity.

\subsubsection{The quantum plane.}

Let us begin with $A=k[x]$ and $B=k[y]$. In this case, for any $x^{i_{1}} \otimes \cdots \otimes x^{i_{m}} \otimes x^{i} y^{j} \in \mathrm{CH}_{m}\left(A, A \#_{R} B\right)$, we have

$$
\left[y^{j}, x^{i_{1}} \otimes \cdots \otimes x^{i_{m}} \otimes x^{i}\right]=\left(q^{\left(i_{1}+\ldots+i_{m}+i\right) j}-1\right) x^{i_{1}} \otimes \cdots \otimes x^{i_{m}} \otimes x^{i} y^{j},
$$

that is,

$$
\mathrm{CH}_{m}\left(A, A \#_{R} B\right)_{B} \cong \mathrm{CH}_{m}(k, B / k) \oplus \mathrm{CH}_{m}(A)
$$

whereas,

$$
\mathrm{CH}_{m}\left(A, A \#_{R} B\right)^{B} \cong \mathrm{CH}_{m}(k, B) .
$$

Accordingly, we see that

$$
\begin{aligned}
& H H_{n}\left(A \#_{R} B\right) \cong H_{n}\left(\mathrm{CH}_{*}\left(A, A \#_{R} B\right)_{B}\right) \oplus H_{n-1}\left(\mathrm{CH}_{*}\left(A, A \#_{R} B\right)^{B}\right) \\
& \cong \begin{cases}k[x] \oplus y k[y] & \text { if } n=0, \\
k[x] \oplus k[y] & \text { if } n=1, \\
0 & \text { if } n \geqslant 2 .\end{cases}
\end{aligned}
$$

\subsubsection{The quantum cylinder.}

We now consider $A=k[x]$ and $B=k\left[y, y^{-1}\right]$. In this case, for any $x^{i_{1}} \otimes \cdots \otimes x^{i_{m}} \otimes x^{i} y^{j} \in \mathrm{CH}_{m}\left(A, A \#_{R} B\right)$ so that $i_{1}+\ldots+i_{m}+i \neq 0$, we may choose $j_{1}, j_{2} \in \mathbb{Z}$ such that $j_{1}+j_{2}=j$ and that $j_{1} \neq 0$ to get

$$
\left[y^{j_{1}}, x^{i_{1}} \otimes \cdots \otimes x^{i_{m}} \otimes x^{i} y^{j_{2}}\right]=\left(q^{\left(i_{1}+\ldots+i_{m}+i\right) j_{1}}-1\right) x^{i_{1}} \otimes \cdots \otimes x^{i_{m}} \otimes x^{i} y^{j},
$$

that is,

$$
\mathrm{CH}_{m}\left(A, A \#_{R} B\right)_{B} \cong \mathrm{CH}_{m}(k, B) \cong \mathrm{CH}_{m}\left(A, A \#_{R} B\right)^{B} .
$$

Accordingly, we see that

$$
H H_{n}\left(A \#_{R} B\right) \cong \begin{cases}k\left[y, y^{-1}\right] & \text { if } n=0,1 \\ 0 & \text { if } n \geqslant 2 .\end{cases}
$$




\subsubsection{The quantum torus.}

We continue with $A=k\left[x, x^{-1}\right]$ and $B=k\left[y, y^{-1}\right]$. In this case, for any $x^{i_{1}} \otimes \cdots \otimes x^{i_{m}} \otimes x^{i} y^{j} \in$ $\mathrm{CH}_{m}\left(A, A \#_{R} B\right)$ so that $i_{1}+\ldots+i_{m}+i \neq 0$, we may choose $j_{1}, j_{2} \in \mathbb{Z}$ so that $j_{1}+j_{2}=j$ and that $j_{1} \neq 0$ to get

$$
\left[y^{j_{1}}, x^{i_{1}} \otimes \cdots \otimes x^{i_{m}} \otimes x^{i} y^{j_{2}}\right]=\left(q^{\left(i_{1}+\ldots+i_{m}+i\right) j_{1}}-1\right) x^{i_{1}} \otimes \cdots \otimes x^{i_{m}} \otimes x^{i} y^{j}
$$

that is,

$$
\mathrm{CH}_{m}\left(A, A \#_{R} B\right)_{B}=\mathrm{CH}_{m}\left(A, A \#_{R} B\right)^{B} \cong\left\langle x^{i_{1}} \otimes \cdots \otimes x^{i_{m}} \otimes x^{i} y^{j} \mid i_{1}+\ldots+i_{m}+i=0\right\rangle,
$$

Just like we did in Lemma we next identify the above complexes with the complex computing the homology of the group algebra $k[\mathbb{Z}]$ with coefficients in $k\left[y, y^{-1}\right]$ via

$$
\begin{aligned}
\mathrm{CH}_{n}\left(A, A \#_{R} B\right)_{B} \cong & \mathrm{CH}_{n}\left(A, A \#_{R} B\right)^{B} \rightarrow \mathrm{CH}_{n}\left(k[\mathbb{Z}], k\left[y, y^{-1}\right]\right), \\
& x^{\ell_{1}} \otimes \cdots \otimes x^{\ell_{n}} \otimes\left(x^{-\ell_{1}-\ldots-\ell_{n}} \otimes y^{j}\right) \mapsto \ell_{1} \otimes \cdots \otimes \ell_{n} \otimes y^{j},
\end{aligned}
$$

where the right $\mathbb{Z}$-module structure on $k\left[y, y^{-1}\right]$ is given by $y^{j} \triangleleft i:=q^{i j}$, and the left $\mathbb{Z}$-module structure is trivial. As such,

$$
\begin{gathered}
H H_{n}\left(A \#_{R} B\right) \cong H_{n}\left(\mathrm{CH}_{*}\left(A, A \#_{R} B\right)_{B}\right) \oplus H_{n-1}\left(\mathrm{CH}_{*}\left(A, A \#_{R} B\right)^{B}\right) \cong \\
H_{n}\left(k[\mathbb{Z}], k\left[y, y^{-1}\right]\right) \oplus H_{n-1}\left(k[\mathbb{Z}], k\left[y, y^{-1}\right]\right) .
\end{gathered}
$$

It is then evident, in view of [4, Ex.III.1.1], that

$$
H H_{n}\left(A \#_{R} B\right) \cong \begin{cases}k & \text { if } n=0 \\ k \oplus k & \text { if } n=1 \\ 0 & \text { if } n \geqslant 2\end{cases}
$$

\subsection{The multiparametric affine space.}

The multiparametric affine space $S\left(X_{v}, \Lambda\right)$ defined in [16, Subsect. 3.1] is the algebra generated by $X_{v}:=\left\{x_{1}, \ldots, x_{v}\right\}$ subject to the relations

$$
x_{j} x_{i}=q_{i, j} x_{i} x_{j},
$$

where for $1 \leqslant i, j \leqslant v$, and the structure constants $\Lambda:=\left(q_{i, j}\right)$ form a set of nonzero elements in $k$ satisfying

$$
q_{i, i}=1, \quad q_{i, j} q_{j, i}=1,
$$

for all $i<j$. For the sake of simplicity, we are going to drop $\Lambda$ from the notation.

Now, we can write $S\left(X_{v}\right)$ as an iterated sequence of smash biproducts

$$
S\left(X_{v}\right)=\underbrace{k\left[x_{1}\right] \# \cdots \# k\left[x_{n}\right]}_{v \text {-times }}=S\left(X_{v-1}\right) \# k\left[x_{v}\right]
$$

In view of Theorem 2.7, we have

$$
H H_{n}\left(S\left(X_{v}\right)\right) \cong H_{n}\left(\mathrm{CH}_{*}\left(S\left(X_{v-1}\right), S\left(X_{v}\right)\right)_{k\left[x_{v}\right]}\right) \oplus H_{n-1}\left(\mathrm{CH}_{*}\left(S\left(X_{v-1}\right), S\left(X_{v}\right)\right)^{k\left[x_{v}\right]}\right) .
$$




\subsubsection{The free case.}

Now, let us assume for $1 \leqslant i, j \leqslant v$ that $\Lambda$ generates a free abelian group of rank $v(v-1) / 2$ in $k^{\times}$. Then,

$$
\mathrm{CH}_{*}\left(S\left(X_{v-1}\right), S\left(X_{v}\right)\right)_{k\left[x_{v}\right]} \cong \mathrm{CH}_{*}\left(S\left(X_{v-1}\right), S\left(X_{v-1}\right)\right) \text {, }
$$

and

$$
\mathrm{CH}_{*}\left(S\left(X_{v-1}\right), S\left(X_{v}\right)\right)^{k\left[x_{v}\right]} \cong \mathrm{CH}_{*}\left(k, k\left[x_{v}\right]\right) .
$$

It is then immediate to see that

$$
H H_{n}\left(S\left(X_{v}\right)\right) \cong \begin{cases}k \oplus \bigoplus_{i=1}^{v} x_{i} k\left[x_{i}\right] & \text { if } n=0 \\ \bigoplus_{i=1}^{v} k\left[x_{i}\right] \otimes x_{i} & \text { if } n=1 \\ 0 & \text { if } n \geqslant 2\end{cases}
$$

which is the case $r=0$ in [26, Thm. 4.4.1].

\subsubsection{The non-free case.}

Hochschild complex admits the $\mathbb{N}^{v}$-grading on $S\left(X_{\nu}, \Lambda\right)$ where we have

$$
H H_{*}\left(S\left(X_{\nu}\right)\right)=\bigoplus_{\left(m_{1}, \ldots, m_{\nu}\right) \in \mathbb{N}^{v}} H H_{*}^{\left(m_{1}, \ldots, m_{\nu}\right)}\left(S\left(X_{\nu}\right)\right)
$$

The same grading applies to both $\mathrm{CH}_{*}\left(S\left(X_{v-1}\right), S\left(X_{v}\right)\right)_{k\left[x_{\nu}\right]}$ and $\mathrm{CH}_{*}\left(S\left(X_{v-1}\right), S\left(X_{v}\right)\right)^{k\left[x_{\nu}\right]}$. Then it suffices to consider only the components with multi-degree $\left(m_{1}, \ldots, m_{v}\right) \in \mathbb{N}^{v}$.

Now, consider the cone $C$ in the lattice $\mathbb{N}^{v}$ of elements $\left(m_{1}, \ldots, m_{v}\right) \in \mathbb{N}^{v}$ with the property that

$$
\prod_{i=1}^{v} q_{j, i}^{m_{i}}=1
$$

for every $1 \leqslant j \leqslant v$. This translates into

$$
x_{j} \cdot\left(x_{1}^{m_{1}} \ldots x_{v}^{m_{v}}\right)=\left(x_{1}^{m_{1}} \ldots x_{v}^{m_{v}}\right) \cdot x_{j}
$$

for $1 \leqslant j \leqslant v$. Hence, it follows at once from (3.4) and (3.5) that

$$
\operatorname{dim}_{k} H H_{n}^{\left(m_{1}, \ldots, m_{\nu}\right)}\left(S\left(X_{v}\right)\right)= \begin{cases}\left(h\left(m_{1}, \ldots, m_{\nu}\right)\right. & n \\ 0 & \text { if }\left(m_{1}, \ldots, m_{v}\right) \in C\end{cases}
$$

where we define

$$
h\left(m_{1}, \ldots, m_{v}\right)=\#\left\{m_{i} \mid m_{i}>0\right\},
$$

i.e. it counts the number of $m_{i}>0$. Our result agrees with [16, Thm. 3.1.1], and also with [30, Thm. 6.1].

\subsection{Quantum complete intersections.}

Given two integers $a, b \geqslant 2$, and $q \in k$ which is not a root of unity; let $C_{a, b}$ be the quotient of the algebra $k\{x, y\}$ generated by two noncommuting indeterminates divided by the two sided ideal given by the relations

$$
x^{a}, \quad y x-q x y, \quad y^{b}
$$

as given in [3]. We note that the choice of $a=b=2$ yields the quantum exterior algebra [2]. A linear basis of this algebra is given by monomials of the form $x^{i} y^{j}$ where $0 \leqslant i<a$ and $0 \leqslant j<b$.

Now, consider the truncated polynomial algebras $T_{a}:=k[x] /\left\langle x^{a}\right\rangle$, and $T_{b}:=k[y] /\left\langle y^{b}\right\rangle$ determined by $a, b \geqslant 2$. Then

$$
R: T_{b} \otimes T_{a} \rightarrow T_{a} \otimes T_{b} ; \quad R\left(y^{j} \otimes x^{i}\right):=q^{i j} x^{i} \otimes y^{j},
$$

an invertible distributive law. Thus we have $C_{a, b} \cong T_{a} \#_{R} T_{b}$. 
The Hochschild homology of a truncated polynomial algebra $T_{a}:=k[x] /\left\langle x^{a}\right\rangle$ has the periodic resolution $\left(P_{*}, \partial_{*}\right)$ where $P_{n}=T_{a} \otimes T_{a}$ and

$$
\partial_{n}= \begin{cases}1 \otimes x-x \otimes 1 & \text { if } n \text { is odd } \\ \sum_{i=0}^{a-1} x^{i} \otimes x^{a-1-i} & \text { if } n \text { is even. }\end{cases}
$$

for $n \geqslant 1$ [19, Sect. 5.9], see also [20, E.4.1.8]. Tensoring (over $T_{a} \otimes T_{a}$ ) with a $T_{a}$-bimodule $U$, then $H_{n}\left(T_{a}, U\right)$ appears as the homology of the complex

$$
\cdots \longrightarrow \stackrel{\partial_{4}}{\longrightarrow} U \stackrel{\partial_{3}}{\longrightarrow} U \stackrel{\partial_{2}}{\longrightarrow} U \stackrel{\partial_{1}}{\longrightarrow} U \longrightarrow 0,
$$

where for $n \geqslant 1$,

$$
\partial_{n}(u)= \begin{cases}x u-u x & \text { if } n \text { is odd } \\ \sum_{i=0}^{a-1} x^{i} u x^{a-1-i} & \text { if } n \text { is even. }\end{cases}
$$

More precisely, for any $m \geqslant 1$ and $x^{i_{1}} \otimes \cdots \otimes x^{i_{m}} \otimes x^{i} y^{j} \in \mathrm{CH}_{m}\left(T_{a}, T_{a} \#_{R} T_{b}\right)$ we have

$$
\partial_{n}\left(x^{i_{1}} \otimes \cdots \otimes x^{i_{m}} \otimes x^{i} y^{j}\right)= \begin{cases}\left(q^{i_{1}+\cdots+i_{m}+i}-1\right) x^{i_{1}} \otimes \cdots \otimes x^{i_{m}} \otimes x^{i} y^{j+1} & \text { if } n \text { is off, } \\ \sum_{s=0}^{b-1} q^{\left(i_{1}+\cdots+i_{m}+i\right) s} x^{i_{1}} \otimes \cdots \otimes x^{i_{m}} \otimes x^{i} y^{b-1+j} & \text { if } n \text { is even. }\end{cases}
$$

We now see that $H H_{q}\left(T_{a}\right) \cong k^{a-1}$ for $q>0$, as in [20, Prop. 5.4.15] Accordingly, by Theorem 2.2] we get

$$
H_{p}\left(T_{b}, \mathrm{CH}_{*}\left(T_{a}, C_{a, b}\right)\right)= \begin{cases}\mathrm{CH}_{*}\left(k, T_{b} / k\right) \oplus \mathrm{CH}_{*}\left(T_{a}\right), & \text { if } p=0, \\ \mathrm{CH}_{*}\left(k, T_{b} /\left\langle y^{b-1}\right\rangle\right) & \text { if } p \text { is odd, } \\ \mathrm{CH}_{*}\left(k, T_{b} / k\right) & \text { if } p>0 \text { is even. }\end{cases}
$$

Then the 1st page of the spectral sequence (2.5) appears as

$$
H H_{*}\left(C_{a, b}\right) E^{\prime} E_{p, q}^{1} \cong \begin{cases}\mathrm{CH}_{q}\left(k, T_{b} / k\right) \oplus \mathrm{CH}_{q}\left(T_{a}\right) & \text { if } p=0, \\ \mathrm{CH}_{q}\left(k, T_{b} /\left\langle y^{b-1}\right\rangle\right) & \text { if } p \text { is odd, } \\ \mathrm{CH}_{q}\left(k, T_{b} / k\right) & \text { if } p>0 \text { is even. }\end{cases}
$$

Finally, since $H_{q}\left(k, T_{b} /\left\langle y^{b-1}\right\rangle\right)=0=H_{q}\left(k, T_{b} / k\right)$ for $q>0$, the vertical homology yields

$$
{ }^{\prime} E_{p, q}^{2} \cong \begin{cases}T_{b} / k \oplus k^{a} & \text { if } p=q=0 \\ k^{a-1} & \text { if } p=0 \text { and } q>0, \\ T_{b} /\left\langle y^{b-1}\right\rangle & \text { if } p \text { is odd and } q=0, \\ T_{b} / k & \text { if } p>0 \text { is even and } q=0, \\ 0 & \text { otherwise. }\end{cases}
$$

In other words,

$$
\operatorname{dim}_{k} H H_{n}\left(C_{a, b}\right)= \begin{cases}a+b-1 & \text { if } n=0 \\ a+b-2 & \text { if } n \geqslant 1\end{cases}
$$

as in [3, Thm. 3.1].

\subsection{The deformed (completed) Weyl algebras.}

Let $\mathfrak{g}$ be a finite dimensional Lie algebra, and $V$ be a vector space. Let also $S(V)$ denote the symmetric algebra on $V$, and $\widehat{S(V)}$ be the completed symmetric algebra over $V$, i.e. the algebra of formal power series in $\operatorname{dim}(V)$-many variables. We note that the algebra $\widehat{S(V)}$ is isomorphic to the dual algebra $S(V)^{\vee}$ of $S(V)$ viewed as the coalgebra of polynomials over $\operatorname{dim}(V)$-many commuting variables. 
Let us now recall the $(\mathfrak{g}, D)$-deformed Weyl algebras from [9, 21]. Given $D: \mathfrak{g} \rightarrow \operatorname{Hom}(V, \widehat{S(V)})$, extending it to a morphism $D: \mathfrak{g} \rightarrow \operatorname{Der}(\widehat{S(V)})$ of Lie algebras [21, Subsect. 1.2], one arrives at the Hopf-cross product, $A_{\mathfrak{g}, D}:=\widehat{S(V)} \rtimes U(\mathfrak{g})$, called the $(\mathfrak{g}, D)$-deformed (completed) Weyl algebra.

We are going to identify the (continuous) Hochschild homology of the algebra $A_{\mathfrak{g}, D}$ with the homology of its dense subalgebra $A_{\mathfrak{g}, D}^{\mathrm{pol}}:=S(V) \rtimes U(\mathfrak{g})$.

Proposition 3.2. If $D(g)(v) \in \mathbb{R}\langle 1\rangle \oplus V$, for any $g \in \mathfrak{g}$ and any $v \in V$, then there is an isomorphism of the form $H H_{*}\left(A_{\mathrm{g}, D}^{\mathrm{pol}}\right) \cong H H_{*}^{\text {cont }}\left(A_{\mathrm{g}, D}\right)$, where the right hand side refers to the continuous Hochschild homology.

Proof. It follows at once from the hypothesis that, the distributive law between $\widehat{S(V)}$ and $U(\mathfrak{g})$ restricts to a degree preserving distributive law between $S(V)$ and $U(\mathfrak{g})$. As such, we have a subcomplex

$$
\mathrm{CH}_{*}(S(V) \rtimes U(\mathfrak{g}))_{(m)} \subseteq \mathrm{CH}_{*}(S(V) \rtimes U(\mathfrak{g}))
$$

of terms whose total degree in $S(V)$ is less than or equal to $m \in \mathbb{N}$. This collection yields a projective system of complexes together with the natural epimorphisms

$$
\mathrm{CH}_{*}(S(V) \rtimes U(\mathfrak{g}))_{(m+1)} \rightarrow \mathrm{CH}_{*}(S(V) \rtimes U(\mathfrak{g}))_{(m)}
$$

The system satisfies the Mittag-Leffler condition [8, 11] by definition. Then we conclude from [11, Prop. 2 and Thm. 5] that

$$
H H_{*}^{\text {cont }}\left(A_{\mathfrak{g}, D}\right) \cong \underset{\leftarrow}{\lim _{m}} H H_{*}(S(V) \rtimes U(\mathfrak{g}))_{(m)}
$$

where the left hand side is the continuous Hochschild homology of $A_{\mathfrak{g}, D}$.

On the other hand, since the distributive law is degree preserving, the collection $\mathrm{CH}_{*}(S(V) \rtimes U(\mathfrak{g}))_{(m)}$ forms also an injective system of complexes via the natural embeddings

$$
\mathrm{CH}_{*}(S(V) \rtimes U(\mathfrak{g}))_{(m)} \rightarrow \mathrm{CH}_{*}(S(V) \rtimes U(\mathfrak{g}))_{(m+1)},
$$

which results in

$$
\mathrm{CH}_{*}(S(V) \rtimes U(\mathfrak{g}))=\underset{m}{\lim } \mathrm{CH}_{*}(S(V) \rtimes U(\mathfrak{g}))_{(m)} .
$$

Since the homology $H H_{*}(S(V) \rtimes U(\mathfrak{g}))$ is bounded and finite dimensional at every degree, by Corollary 2.3, we see that there is an index $N \in \mathbb{N}$ such that for every $m \geqslant N$ the natural injections in (3.11), and therefore the natural projections in (3.9), induce quasi-isomorphisms. In other words,

$$
H H_{*}(S(V) \rtimes U(\mathfrak{g})) \cong H H_{*}^{\operatorname{cont}}(\widehat{S(V)} \rtimes U(\mathfrak{g}))
$$

as we wanted to show.

In particular, for the Weyl algebra $A_{2 n}^{\mathrm{pol}}$, and its completion $A_{2 n}$, in view of the fact that the Hochschild homology of $A_{2 n}^{\mathrm{pol}}$ is known, see for example [19, Sect. 5.10], we get [12, Thm. 2.1] and [13, Thm. 4] in

$$
H H_{m}^{\text {cont }}\left(A_{2 n}\right) \cong H H_{m}\left(A_{2 n}^{\mathrm{pol}}\right)= \begin{cases}k & \text { if } m=2 n \\ 0 & \text { otherwise }\end{cases}
$$

for any $n \geqslant 1$ and any $m \geqslant 0$, as an immediate corollary to Proposition 3.2 . 


\subsection{Quantum Weyl algebras.}

In this subsection we are going to discuss the Hochschild homology of quantum Weyl algebras $S\left(X_{\mu}, Y_{\nu}, \Lambda\right)$ generated by $X_{\mu}:=\left\{x_{1}, \ldots, x_{\mu}\right\}$ and $Y_{v}:=\left\{y_{1}, \ldots, y_{v}\right\}$, subject to the relations

$$
x_{i} x_{j}-q_{i, j} x_{j} x_{i}, \quad y_{i} y_{j}-q_{i, j} y_{j} y_{i}, \quad y_{j} x_{i}-q_{i, j} x_{i} y_{j}, \quad x_{i} y_{i}-y_{i} x_{i}+1
$$

for all $i \neq j$ where $1 \leqslant i \leqslant \mu, 1 \leqslant j \leqslant v$, and $\Lambda:=\left(q_{i, j}\right)$ of (3.3). Since there is a an isomorphism of algebras of the form $S\left(X_{\mu}, Y_{v}, \Lambda\right) \rightarrow S\left(X_{v}, Y_{\mu}, \Lambda^{t}\right)$ given by

$$
x_{i} \mapsto-y_{i}, \quad y_{j} \mapsto x_{j},
$$

we may assume, without loss of generality, that $v \geqslant \mu$. We also assume that $\Lambda$ generates a free abelian group of rank $v(v-1) / 2$ in $k^{\times}$as in [26]. For simplicity, from now on we are going to drop the parameter matrix $\Lambda$ from the notation.

\subsubsection{The case $\mu=v$.}

Let $v=\mu$, and let $A_{2}\left(x_{i}, y_{i}\right)$ be the copy of the ordinary Weyl algebra in $S\left(X_{\mu}, Y_{\mu}\right)$ generated by $x_{i}$ and $y_{i}$ for $1 \leqslant i, j \leqslant \mu$. We recall also the $A_{2}\left(x_{1}, y_{1}\right)^{e}$-resolution

$$
0 \rightarrow A_{2}\left(x_{1}, y_{1}\right)^{e} \stackrel{\partial_{2}}{\longrightarrow} A_{2}\left(x_{1}, y_{1}\right)^{e} \oplus A_{2}\left(x_{1}, y_{1}\right)^{e} \stackrel{\partial_{1}}{\longrightarrow} A_{2}\left(x_{1}, y_{1}\right)^{e} \rightarrow 0,
$$

where

$$
\partial_{2}(a \otimes b)=\left(a x_{1} \otimes b-a \otimes x_{1} b\right) e_{1}-\left(a y_{1} \otimes b-a \otimes y_{1} b\right) e_{2},
$$

and

$$
\partial_{1}\left(\left(a_{1} \otimes b_{1}\right) e_{1}+\left(a_{2} \otimes b_{2}\right) e_{2}\right)=a_{1} y_{1} \otimes b_{1}-a_{1} \otimes y_{1} b_{1}+a_{2} x_{1} \otimes b_{2}-a_{2} \otimes x_{1} b_{2},
$$

for $A_{2}\left(x_{1}, y_{1}\right)$, [19, Sect. 5.10]. Since $S\left(X_{\mu}, Y_{\mu}\right) \cong S\left(X_{\mu-1}, Y_{\mu-1}\right) \# A_{2}\left(x_{1}, y_{1}\right)$, in view of Theorem 2.2 we have

$$
H H_{*}\left(S\left(X_{\mu}, Y_{\mu}\right)\right) \Leftarrow{ }^{\prime} E_{p, q}^{1}=H_{p}\left(A_{2}\left(x_{1}, y_{1}\right), \mathrm{CH}_{q}\left(S\left(X_{\mu-1}, Y_{\mu-1}\right), S\left(X_{\mu}, Y_{\mu}\right)\right)\right) .
$$

Now we note that

$$
\begin{aligned}
& {\left[y_{1}, x_{2}^{a_{2}} y_{2}^{b_{2}} \otimes \cdots \otimes x_{\mu}^{a_{\mu}} y_{\mu}^{b_{\mu}} \otimes x_{1}^{a} y_{1}^{b}\right]} \\
& =\left(q_{1,2}^{B_{2}-A_{2}} \cdots q_{1, \mu}^{B_{\mu}-A_{\mu}}-1\right) x_{2}^{a_{2}} y_{2}^{b_{2}} \otimes \cdots \otimes x_{\mu}^{a_{\mu}} y_{\mu}^{b_{\mu}} \otimes x_{1}^{a} y_{1}^{b+1} \\
& \quad+a q_{1,2}^{B_{2}-A_{2}} \cdots q_{1, \mu}^{B_{\mu}-A_{\mu}} x_{2}^{a_{2}} y_{2}^{b_{2}} \otimes \cdots \otimes x_{\mu}^{a_{\mu}} y_{\mu}^{b_{\mu}} \otimes x_{1}^{a-1} y_{1}^{b},
\end{aligned}
$$

and that

$$
\begin{aligned}
& {\left[x_{1}, x_{2}^{a_{2}} y_{2}^{b_{2}} \otimes \cdots \otimes x_{\mu}^{a_{\mu}} y_{\mu}^{b_{\mu}} \otimes x_{1}^{a} y_{1}^{b}\right]} \\
& =\left(q_{1,2}^{A_{2}-B_{2}} \cdots q_{1, \mu}^{A_{\mu}-B_{\mu}}-1\right) x_{2}^{a_{2}} y_{2}^{b_{2}} \otimes \cdots \otimes x_{\mu}^{a_{\mu}} y_{\mu}^{b_{\mu}} \otimes x_{1}^{a+1} y_{1}^{b} \\
& \quad+b q_{1,2}^{A_{2}-B_{2}} \cdots q_{1, \mu}^{A_{\mu}-B_{\mu}} x_{2}^{a_{2}} y_{2}^{b_{2}} \otimes \cdots \otimes x_{\mu}^{a_{\mu}} y_{\mu}^{b_{\mu}} \otimes x_{1}^{a} y_{1}^{b-1},
\end{aligned}
$$

where $A_{i}$ and $B_{j}, 2 \leqslant i, j \leqslant \mu$, denote the total degree of $x_{i}$ and $y_{j}$ respectively. Hence, upon tensoring the above resolution with the complex $\mathrm{CH}_{*}\left(S\left(X_{\mu-1}, Y_{\mu-1}\right), S\left(X_{\mu}, Y_{\mu}\right)\right)$ we see that

$$
' E_{p, q}^{1} \cong \begin{cases}\mathrm{CH}_{q}\left(S\left(X_{\mu-1}, Y_{\mu-1}\right), S\left(X_{\mu}, Y_{\mu}\right)\right)^{A_{2}\left(x_{1}, y_{1}\right)} & \text { if } p=2, \\ 0 & \text { otherwise. }\end{cases}
$$

The invariant subcomplex consists of the tensors whose total degree of $x_{i}$ terms is equal to the total degree of $y_{i}$ terms for each $2 \leqslant i \leqslant \mu$, with no $x_{1}$ or $y_{1}$ terms. Moreover, this subcomplex is a direct summand (as differential graded modules) of the Hochschild complex $\mathrm{CH}_{q}\left(S\left(X_{\mu}, Y_{\mu}\right)\right)$, as such, we see that

$$
{ }^{\prime} E_{2, q}^{2} \cong H_{q}\left(\mathrm{CH}_{*}\left(S\left(X_{\mu-1}, Y_{\mu-1}\right), S\left(X_{\mu}, Y_{\mu}\right)\right)^{A_{2}\left(x_{1}, y_{1}\right)}\right)=H H_{q}\left(S\left(X_{\mu-1}, Y_{\mu-1}\right)\right)^{A_{2}\left(x_{1}, y_{1}\right)} .
$$

In other words,

$$
H H_{n}\left(S\left(X_{\mu}, Y_{\mu}\right)\right)=H H_{n-2}\left(S\left(X_{\mu-1}, Y_{\mu-1}\right)\right)^{A_{2}\left(x_{1}, y_{1}\right)} .
$$


Reducing recursively then, we obtain

$$
H H_{n}\left(S\left(X_{\mu}, Y_{\mu}\right)\right) \cong \begin{cases}k & \text { if } n=2 \mu \\ 0 & \text { otherwise. }\end{cases}
$$

3.7.2. The case $v>\mu$.

Considering

$$
S\left(X_{\mu}, Y_{v}\right) \cong S\left(X_{\mu}, Y_{v-1}\right) \#_{R} k\left[y_{v}\right]
$$

Theorem 2.7yields

$$
\begin{aligned}
& H H_{n}\left(S\left(X_{\mu}, Y_{v}\right)\right) \\
& \quad \cong H_{n}\left(\mathrm{CH}_{*}\left(S\left(X_{\mu}, Y_{\nu-1}\right), S\left(X_{\mu}, Y_{v}\right)\right)_{k\left[y_{v}\right]}\right) \oplus H_{n-1}\left(\mathrm{CH}_{*}\left(S\left(X_{\mu}, Y_{\nu-1}\right), S\left(X_{\mu}, Y_{v}\right)\right)^{k\left[y_{\nu}\right]}\right) .
\end{aligned}
$$

It is evident from the commutation relations (3.13) that

$$
\begin{aligned}
& \mathrm{CH}_{*}\left(S\left(X_{\mu}, Y_{v-1}\right), S\left(X_{\mu}, Y_{v}\right)\right)_{k\left[y_{v}\right]} \\
& \cong \mathrm{CH}_{*}\left(S\left(X_{\mu}, Y_{v-1}\right), S\left(X_{\mu}, Y_{v-1}\right)\right) \oplus \mathrm{CH}_{*}\left(k, k\left[y_{v}\right] / k\right) .
\end{aligned}
$$

and that the $k\left[y_{v}\right]$-invariant subcomplex $\mathrm{CH}_{*}\left(S\left(X_{\mu}, Y_{v-1}\right), S\left(X_{\mu}, Y_{v}\right)\right)^{k\left[y_{v}\right]}$ may be written as a direct sum of $\mathrm{CH}_{*}\left(k, k\left[y_{v}\right]\right)$ and a complex with the same total degree of $x_{i}$ 's and $y_{i}$ 's, for each $1 \leqslant i \leqslant \mu$. However, since these complexes are accompanied with an extra $\otimes y_{v}$, the reduction performed in the previous subsection reveals that the homology (on the next page) of the latter is trivial. As a result,

$$
\begin{aligned}
H H_{n}\left(S\left(X_{\mu}, Y_{\nu}\right)\right) \cong & H H_{n}\left(S\left(X_{\mu}, Y_{\nu-1}\right)\right) \oplus H_{n}\left(\mathrm{CH}_{*}\left(k, k\left[y_{\nu}\right] / k\right)\right) \\
& \oplus H_{n-1}\left(\mathrm{CH}_{*}\left(k, k\left[y_{\nu}\right] \otimes y_{\nu}\right)\right) .
\end{aligned}
$$

Hence, inductively, we see that

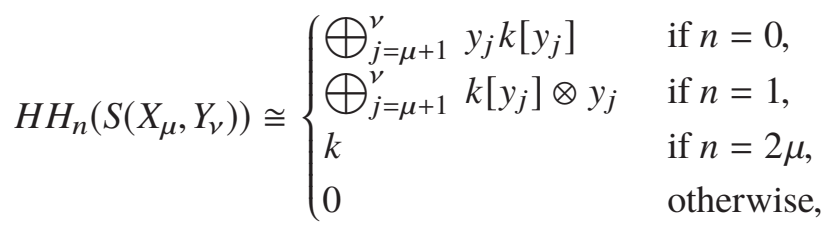

as in [26, Thm. 4.4.1].

\subsection{The algebra of quantum matrices.}

Given $q \neq 1$, the algebra $M_{q}(2)$ of quantum matrices is defined in [18, Def. IV.3.2] as the quotient algebra generated by $\{a, b, c, d\}$ subject to the relations

$$
\begin{array}{rlrl}
b a & =q a b, \\
c a & =q a c, & d b & =q b d, \\
b c & =c b, & a d-d a & =q c d, \\
d c & =\left(q^{-1}-q\right) b c .
\end{array}
$$

We note also that the center of $M_{q}(2)$ is generated by the quantum determinant $D_{q}:=a d-q^{-1} b c \in M_{q}(2)$, see [22, Thm. 1.6]).

More importantly, $M_{q}(2)$ is a tower of Ore extensions $A_{1} \subseteq A_{2} \subseteq A_{3} \subseteq M_{q}(2)$ given by

$$
A_{1}=k[a], \quad A_{2} \cong A_{1}\left[b, \alpha_{1}, 0\right], \quad A_{3} \cong A_{2}\left[c, \alpha_{2}, 0\right], \quad M_{q}(2) \cong A_{3}\left[d, \alpha_{3}, \delta\right],
$$


see, for instance, [18, Sect. IV.4]. The structure morphisms $\alpha_{i}: A_{i} \rightarrow A_{i}$ are given as

$$
\begin{array}{lll}
\alpha_{1}(a):=q a, & & \\
\alpha_{2}(a):=q a, & \alpha_{2}(b):=b, & \\
\alpha_{3}(a):=a, & \alpha_{3}(b):=q b, & \alpha_{3}(c):=q c .
\end{array}
$$

for $i=1,2,3$, and $\delta: A_{3} \rightarrow A_{3}$ is given as

$$
\delta\left(b^{j} c^{k}\right):=0 \quad \delta\left(a^{i} b^{j} c^{k}\right):=\left(q-q^{-1}\right) \frac{1-q^{2 i}}{1-q^{2}} a^{i-1} b^{j+1} c^{k+1} .
$$

Let us also recall from [14, Lemma 1] the relations

$$
d^{n} a=a d^{n}-q\left(1-q^{-2 n}\right) b c d^{n-1}, \quad d a^{n}=a^{n} d+q\left(q^{-2 n}-1\right) a^{n-1} b c .
$$

It follows from Theorem 2.2 that

$$
H H_{*}\left(M_{q}(2)\right) \Leftarrow E_{i, j}^{1}:=H_{j}\left(A_{3}, \mathrm{CH}_{i}\left(k[d], M_{q}(2)\right)\right),
$$

where, setting $U_{i}:=\mathrm{CH}_{i}\left(k[d], M_{q}(2)\right)$, the same results yields also

$$
H_{*}\left(A_{3}, U_{i}\right) \Leftarrow{ }^{\prime} E_{m, n}^{1}:=H_{m}\left(k[c], \mathrm{CH}_{n}\left(A_{2}, U_{i}\right)\right)= \begin{cases}\mathrm{CH}_{n}\left(A_{2}, U_{i}\right)_{k[c]} & \text { if } m=0, \\ \mathrm{CH}_{n}\left(A_{2}, U_{i}\right)^{k[c]} \otimes c & \text { if } m=1, \\ 0 & \text { if } m \geqslant 2 .\end{cases}
$$

On the next step we note that

$$
\left[k[c], M_{q}(2)\right]=\left\langle a^{r} b^{s} c^{t} d^{\ell} \mid r \neq \ell, t>0\right\rangle,
$$

and hence,

$$
\frac{M_{q}(2)}{\left[k[c], M_{q}(2)\right]}:=\left\langle a^{r} b^{s} d^{\ell}, a^{\ell} b^{s} c^{t} d^{\ell} \mid r, s, \ell \geqslant 0, \quad t>0\right\rangle .
$$

Following the ideas of Proposition 2.1 we may identify the $\left(A_{2}\right.$, or vertical) homology of the complex

$$
\begin{aligned}
& \mathrm{CH}_{n}\left(A_{2}, U_{i}\right)_{k[c]} \\
& \cong\left\langle a^{r_{1}} b^{s_{1}} \otimes \cdots \otimes a^{r_{n}} b^{s_{n}} \otimes a^{r_{n+1}} b^{s_{n+1}} d^{\ell_{1}} \otimes d^{\ell_{2}} \otimes \cdots \otimes d^{\ell_{i+1}} \mid r_{u}, s_{v}, \ell_{w} \geqslant 0\right\rangle \\
& \oplus\left\langle a^{r_{1}} b^{s_{1}} \otimes \cdots \otimes a^{r_{n}} b^{s_{n}} \otimes a^{r_{n+1}} b^{s_{n+1}} c^{t} d^{\ell_{1}} \otimes d^{\ell_{2}} \otimes \cdots \otimes d^{\ell_{i+1}}\right| \\
& \left.\quad r_{u}, s_{v}, \ell_{w} \geqslant 0, t>0, \sum_{u} r_{u}=\sum_{w} \ell_{w}\right\rangle
\end{aligned}
$$

with the total homology of the bicomplex

$$
\begin{aligned}
& \mathrm{CH}_{\alpha, \beta}\left(A_{2}, U_{i}\right)_{k[c]} \\
& \cong\left\langle a^{r_{1}} \otimes \cdots \otimes a^{r_{\beta}} \otimes a^{r_{\beta+1}} b^{s} d^{\ell_{1}} \otimes d^{\ell_{2}} \otimes \cdots \otimes d^{\ell_{i+1}} \otimes b^{s_{1}} \otimes \cdots \otimes b^{s_{\alpha}} \mid r_{u}, s, \ell_{w} \geqslant 0\right\rangle \\
& \quad \oplus a^{r_{1}} \otimes \cdots \otimes a^{r_{\beta}} \otimes a^{r_{\beta+1}} b^{s} c^{t} d^{\ell_{1}} \otimes d^{\ell_{2}} \otimes \cdots \otimes d^{\ell_{i+1}} \otimes b^{s_{1}} \otimes \cdots \otimes b^{s_{\alpha}} \mid \\
& \left.\quad r_{u}, s, t, \ell_{w} \geqslant 0, t>0, \sum_{u} r_{u}=\sum_{w} \ell_{w}\right\rangle .
\end{aligned}
$$


The homology of this bicomplex, in turn, is approximated by the spectral sequence

$$
H_{*}\left(\mathrm{CH}_{*}\left(A_{2}, U_{i}\right)_{k[c]}\right)=H_{*}\left(\mathrm{CH}_{* * *}\left(A_{2}, U_{i}\right)_{k[c]}\right)
$$

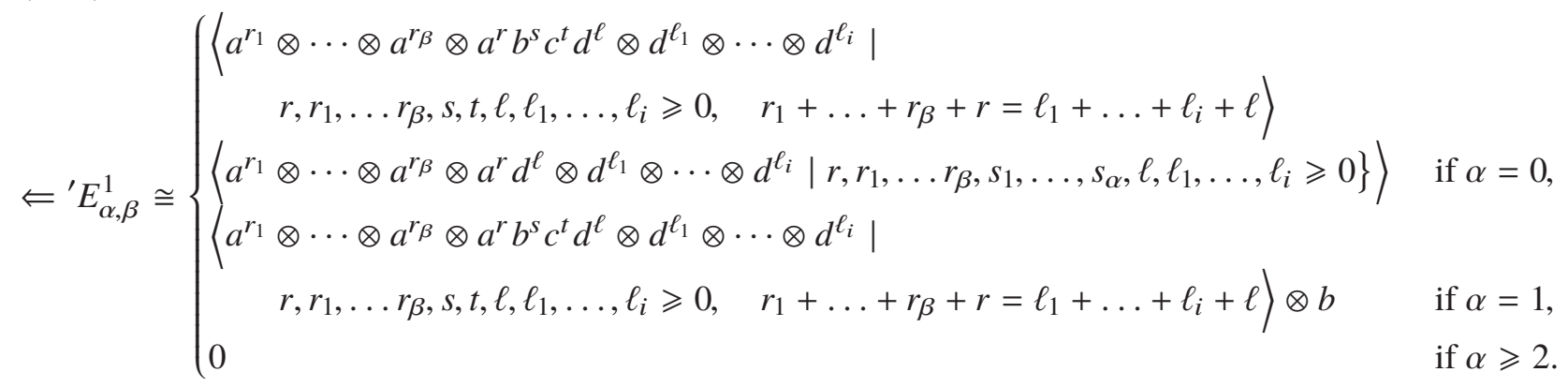

Noticing, in view of 3.15, that

$$
\left[a, a^{\ell-1} b^{s} c^{t} d^{\ell}\right]=\left(1-q^{s+t}\right) a^{\ell} b^{s} c^{t} d^{\ell}-q\left(1-q^{-2 \ell}\right) a^{\ell-1} b^{s+1} c^{t+1} d^{\ell-1},
$$

in the vertical homology we arrive at

$$
\begin{aligned}
& H_{*}\left(\mathrm{CH}_{*}\left(A_{2}, U_{i}\right)_{k[c]}\right) \\
& \Leftarrow^{\prime} E_{\alpha, \beta}^{2} \cong \begin{cases}\mathrm{CH}_{i}(k, k[b, c]) \oplus \mathrm{CH}_{i}(k[d], k[a, d]) & \text { if } \alpha=0, \beta=0, \\
a \otimes \mathrm{CH}_{i}(k, k[a]) & \text { if } \alpha=0, \beta=1 \\
\mathrm{CH}_{i}(k, k[b, c]) \otimes b & \text { if } \alpha=1, \beta=0, \\
0 & \text { otherwise. }\end{cases}
\end{aligned}
$$

Now, on the other hand,

as such,

$$
M_{q}(2)^{k[c]} \cong\left\langle\left\{a^{r} b^{s} c^{t} d^{r} \mid r, s, t \geqslant 0\right\}\right\rangle,
$$

$$
\begin{aligned}
\mathrm{CH}_{n}\left(A_{2}, U_{i}\right)^{k[c]} \cong\left\langle a^{r_{1}} b^{s_{1}} \otimes \cdots \otimes a^{r_{n}} b^{s_{n}} \otimes a^{r} b^{s} c^{t} d^{\ell} \otimes d^{\ell_{1}} \otimes \cdots \otimes d^{\ell_{i}}\right| \\
\left.\quad r, r_{1}, \ldots r_{n}, s, s_{1}, \ldots, s_{n}, t, \ell, \ell_{1}, \ldots, \ell_{i} \geqslant 0, \quad r_{1}+\ldots+r_{n}+r=\ell_{1}+\ldots+\ell_{i}+\ell\right\rangle .
\end{aligned}
$$

Similarly above, we may identify the ( $A_{2}$, or vertical) homology of this complex with the total homology of the bicomplex

$$
\begin{aligned}
& \mathrm{CH}_{\mu, \nu}\left(A_{2}, U_{i}\right)^{k[c]} \cong\left\langle a^{r_{1}} \otimes\right. \cdots \otimes a^{r_{\nu}} \otimes a^{r} b^{s} c^{t} d^{\ell} \otimes d^{\ell_{1}} \otimes \cdots \otimes d^{\ell_{i}} \otimes b^{s_{1}} \otimes \cdots \otimes b^{s_{\mu}} \\
&\left.r, r_{1}, \ldots r_{\nu}, s, s_{1}, \ldots, s_{\mu}, t, \ell, \ell_{1}, \ldots, \ell_{i} \geqslant 0, \quad r_{1}+\ldots+r_{v}+r=\ell_{1}+\ldots+\ell_{i}+\ell\right\rangle .
\end{aligned}
$$

Now, following the same line of thought in 3.16) and (3.17), we obtain

$$
\begin{aligned}
H_{*}\left(\mathrm{CH}_{*}\left(A_{2}, U_{i}\right)^{k[c]} \otimes c\right) & \models^{\prime} E_{\alpha, \beta}^{2} \\
& \cong \begin{cases}\mathrm{CH}_{i}(k, k[b, c]) \otimes c & \text { if } \alpha=0, \beta=0, \\
\mathrm{CH}_{i}(k, k[b, c]) \otimes b \otimes c & \text { if } \alpha=1, \beta=0, \\
0 & \text { otherwise. }\end{cases}
\end{aligned}
$$

We thus conclude,

$$
H_{*}\left(A_{3}, U_{i}\right) \models^{\prime} E_{m, n}^{2} \cong \begin{cases}\mathrm{CH}_{i}(k, k[b, c]) \oplus \mathrm{CH}_{i}(k[d], k[a, d]) & \text { if } m=0 \text { and } n=0, \\ a \otimes \mathrm{CH}_{i}(k, k[a]) \oplus \mathrm{CH}_{i}(k, k[b, c]) \otimes b & \text { if } m=0 \text { and } n=1, \\ \mathrm{CH}_{i}(k, k[b, c]) \otimes c & \text { if } m=1 \text { and } n=0, \\ \mathrm{CH}_{i}(k, k[b, c]) \otimes b \otimes c & \text { if } m=1 \text { and } n=1, \\ 0 & \text { otherwise. }\end{cases}
$$


As such,

$$
\begin{aligned}
H H_{*}\left(M_{q}(2)\right) & \models E_{i, j}^{1} \\
& :=H_{j}\left(A_{3}, \mathrm{CH}_{i}\left(k[d], M_{q}(2)\right)\right) \\
& \cong \begin{cases}\mathrm{CH}_{i}(k, k[b, c]) \oplus \mathrm{CH}_{i}(k[d], k[a, d]) & \text { if } j=0, \\
a \otimes \mathrm{CH}_{i}(k, k[a]) \oplus \mathrm{CH}_{i}(k, k[b, c]) \otimes b \oplus \mathrm{CH}_{i}(k, k[b, c]) \otimes c & \text { if } j=1, \\
\mathrm{CH}_{i}(k, k[b, c]) \otimes b \otimes c & \text { if } j=2, \\
0 & \text { if } j \geqslant 3,\end{cases}
\end{aligned}
$$

and

$$
\begin{aligned}
H H_{*}\left(M_{q}(2)\right) & =E_{i, j}^{2} \\
& \cong \begin{cases}k[b, c] \oplus k[a, d] & \text { if } j=0 \text { and } i=0, \\
k[d] \otimes d & \text { if } j=0 \text { and } i=1, \\
a \otimes k[a] \oplus k[b, c] \otimes b \oplus k[b, c] \otimes c & \text { if } j=1 \text { and } i=0, \\
k[b, c] \otimes b \otimes c & \text { if } j=2 \text { and } i=0, \\
0 & \text { if } j \geqslant 3 \text { or } i \geqslant 1 .\end{cases}
\end{aligned}
$$

In other words, we obtain

$$
H H_{n}\left(M_{q}(2)\right) \cong \begin{cases}k[b, c] \oplus k[a, d] & \text { if } n=0, \\ k[a] \oplus k[b, c] \oplus k[b, c] \oplus k[d] & \text { if } n=1, \\ k[b, c] & \text { if } n=2, \\ 0 & \text { if } n \geqslant 3 .\end{cases}
$$

Remark 3.3. We note that the result above does not follow [16, Coroll. 2.5] since the extension $M_{q}(2)=A_{3}\left[d, \alpha_{3}, \delta\right]$ does not satisfy the hypothesis therein.

\section{REFERENCES}

[1] M. Artin, W. Schelter, and J. Tate. Quantum deformations of GL . Comm. Pure Appl. Math., 44(8-9):879-895, 1991.

[2] P. A. Bergh. On the Hochschild (co)homology of quantum exterior algebras. Comm. Algebra, 35(11):3440-3450, 2007.

[3] P.A. Bergh and K. Erdmann. Homology and cohomology of quantum complete intersections. Algebra Number Theory, 2(5):501-522, 2008.

[4] K. Brown. Cohomology of Groups. Number 87 in Graduate Texts in Mathematics. Springer Verlag, 1982.

[5] S. Caenepeel, B. Ion, G. Militaru, and S. Zhu. The factorization problem and the smash biproduct of algebras and coalgebras. Algebr. Represent. Theory, 3(1):19-42, 2000.

[6] A. Cap, H. Schichl, and J. Vanžura. On twisted tensor products of algebras. Comm. Algebra, 23(12):4701-4735, 1995.

[7] J. Cuntz and D. Quillen. Algebra extensions and nonsingularity. Journal of the American Mathematical Society, 8(2):251289, 1995.

[8] R. M. Dimitrić. A note on surjective inverse systems. Int. J. Pure Appl. Math., 10(3):349-356, 2004.

[9] N. Durov, S. Meljanac, A. Samsarov, and Z. Škoda. A universal formula for representing Lie algebra generators as formal power series with coefficients in the Weyl algebra. J. Algebra, 309(1):318-359, 2007.

[10] S. Eilenberg and S. MacLane. Homology theories for multiplicative systems. 71:294-330, 1951.

[11] I. Emmanouil. Mittag-Leffler condition and the vanishing of $\lim ^{1}$. Topology, 35(1):267-271, 1996.

[12] B. Feigin, G. Felder, and B. Shoikhet. Hochschild cohomology of the Weyl algebra and traces in deformation quantization. Duke Math. J., 127(3):487-517, 2005.

[13] B. L. Feigin and B. L. Tsygan. Cohomology of Lie algebras of generalized Jacobi matrices. Funktsional. Anal. i Prilozhen., 17(2):86-87, 1983

[14] M. Ge, X. Liu, and C. Sun. Representations of quantum matrix algebra $M_{q}(2)$ and its $q$-boson realization. J. Math. Phys., 33(7):2541-2545, 1992.

[15] J. Gómez Torrecillas and T. H. Lenagan. Poincaré series of multi-filtered algebras and partitivity. J. London Math. Soc. (2), 62(2):370-380, 2000.

[16] J. A. Guccione and J. J. Guccione. Hochschild and cyclic homology of Ore extensions and some examples of quantum algebras. K-Theory, 12(3):259-276, 1997.

[17] B. E. Johnson. Cohomology in Banach algebras. American Mathematical Society, 1972.

[18] C. Kassel. Quantum groups, volume 155 of Graduate Texts in Mathematics. Springer-Verlag, 1995. 
[19] C. Kassel. Homology and cohomology of associative algebras. A concise introduction to cyclic homology. École thématique. Août 2004 à ICTP, Trieste (Italie), 2006.

[20] J. L. Loday. Cyclic homology, volume 301 of Grundlehren der Mathematischen Wissenschaften [Fundamental Principles of Mathematical Sciences]. Springer-Verlag, second edition, 1998. Appendix E by Maria O. Ronco, Chapter 13 by the author in collaboration with Teimuraz Pirashvili.

[21] S. Meljanac and Z. Škoda. Leibniz rules for enveloping algebras in symmetric ordering. arXiv:0711.0149v2, 2007.

[22] M. Noumi, H. Yamada, and K. Mimachi. Finite-dimensional representations of the quantum group $\mathrm{GL}_{q}(n ; \mathbf{C})$ and the zonal spherical functions on $\mathrm{U}_{q}(n-1) \backslash \mathrm{U}_{q}(n)$. Japan. J. Math. (N.S.), 19(1):31-80, 1993.

[23] B. Parshall and J.P. Wang. Quantum linear groups. Mem. Amer. Math. Soc., 89(439):vi+157, 1991.

[24] J.-P. Pier. Amenable locally compact groups. Wiley, 1984.

[25] N. Yu. Reshetikhin, L. A. Takhtadzhyan, and L. D. Faddeev. Quantization of Lie groups and Lie algebras. Algebra i Analiz, 1(1):178-206, 1989.

[26] L. Richard. Hochschild homology and cohomology of some classical and quantum noncommutative polynomial algebras. Journal of Pure and Applied Algebra, 187(1):255 - 294, 2004.

[27] W. F. Schelter. Smooth algebras. Journal of Algebra, 103(2):677-685, 1986.

[28] S.F. Siegel and S.J. Witherspoon. The Hochschild cohomology ring of a group algebra. Proc. London Math. Soc., 79 (3)(1):131-157, 1999.

[29] M. van den Bergh. A relation between Hochschild homology and cohomology for Gorenstein rings. Proc. Amer. Math. Soc., 126(5):1345-1348, 1998.

[30] M. Wambst. Complexes de Koszul quantiques. Ann. Inst. Fourier (Grenoble), 43(4):1089-1156, 1993.

Istanbul Technical University, Istanbul, Turkey

E-mail address: kaygun@itu.edu.tr

IŞIK UNIVERsity, ISTANBUL, TURKEY

E-mail address: serkan.sutlu@isikun.edu.tr 